\title{
Editorial
}

\section{Reviewing the future of the p53 field}

\author{
Arnold J Levine ${ }^{\star, 1}$ \\ Cell Death and Differentiation (2018) 25, 1-2; doi:10.1038/cdd.2017.181
}

Fifty years ago, when the authors of the reviews published here were in various stages of training for a future in medical research, the causes of cancers in humans were, at best, enigmatic. We knew that some viruses could cause cancers, some chemicals could cause cancers, and genetic predispositions could be observed for some cancers. But just what the relationships were among viruses, chemicals, and genes were matters of heated debate. The decade of the 1970s uncovered cellular oncogenes captured in viruses, the role of chemical mutagens in converting proto-oncogenes to oncogenes, and the discoveries of tumor suppressor genes (retinoblastoma, p53). As it became clear that genetic mutations, and even a few viruses, could cause cancers in animals and humans, defects in DNA repair processes were shown to contribute to a higher mutation rate and inherited and spontaneous cancers in humans. These hard won facts became the foundations of oncology. What has been surprising is how many different genes in the human genome, altered by mutations, contribute to the formation of cancers over a lifetime. Different cancers have very heterogeneous genetic defects, even when they are of the same tissue and tumor types. But, not all oncogenes, tumor suppressor genes, or DNA repair defects, are created or function equally, nor do they contribute equally to the origins and propagation of cancers.

All of the genomic studies searching for common features in thousands of different cancers conclude that about $50 \%$ of all cancers have Tp53 mutations and exclude all copies of the wild-type Tp53 gene. In addition, a variable number of cancers inactivate $\mathrm{p} 53$ functions by amplifying or overexpressing the negative regulators of p53 (MDM-2, WIP-1). Rather clearly, the most common feature of cancers in humans is the loss of some functions of the wild-type Tp53 gene. That is the justification for, and the importance of, the reviews presented here. But, more important, the nine reviews presented here do not look back over the 38 years of research about the p53 protein and the Tp53 gene. Instead, these reviews address unanswered questions, providing a background, and elucidating the paths to answers that will come over the next few years. This collection of manuscripts reviews the future.

Although Tp53 mutations are the most common feature of human cancers, the nature of the Tp53 mutations (mostly missense mutations) and the mutational alleles observed in the p53 gene of many cancer types, are not uniform. Rather, there are hot spot missense mutations, where mutations at 10 codons (out of 190 different mutations observed in the p53 DNA-binding domain) account for $33 \%$ of all the p53 mutations found in cancers (reviewed in Baugh et al. ${ }^{1}$ ). One reason for this is the presence of methylated $\mathrm{CpG}$ dinucleotides in the Tp53 gene at 7 of these 10 hot spot mutations where methylated CpG dinucleotides have a 10-fold higher mutation frequency than unmethylated CpGs. Indeed, C to T transitions that result from the presence of methylated $\mathrm{CpG}$ dinucleotides are the most common point mutations observed in all human cancers at many different genetic loci. In addition, these seven Tp53 codons encode arginines that are conserved over large evolutionary time spans in the Tp53 genes, and so changes at these sites might be expected to contribute to a loss of function. ${ }^{1}$ There is some evidence that these alleles are selected for in tumors. One reason why only some mutant alleles are selected for in a tumor is the 'gain of function(s)' that some missense mutant alleles confer upon tumors (reviewed in Kim et al. ${ }^{2}$ ). What are the mechanisms that mediate a gain of a function in the p53 mutant protein, and do different mutant alleles produce proteins with different mechanisms for this gain of function? Do diverse tumor tissue types influence the properties of a gain of function? Do the advantages of a gain of function change with the stage of a tumor, the treatment protocols employed, or the other mutations in cancer cells? ${ }^{2}$ These questions need to be answered if we are to understand the biology of the $p 53$ gene and its protein.

The wild-type $\mathrm{p} 53$ protein is a transcription factor that selectively regulates the transcriptional activation of $>200$ genes in diverse cells (reviewed in Engeland ${ }^{3}$ and Sullivan et al. ${ }^{4}$ ). Activation of p53-mediated transcription by various stress signals results in the expression of a number of pathways that accomplish diverse phenotypes that include cell cycle arrest, ${ }^{3}$ senescence, apoptosis and other types of cell death, metabolic alterations, DNA repair processes, and other prosurvival mechanisms and tumor suppression (reviewed in refs. 3-7). There is an active debate about which p53-regulated genes and pathways are responsible for the tumor suppressor phenotype. 5,6 The answer to this question may reside in a better understanding of how stress activates the p53 protein and how a particular stress initiates a transcriptional pathway resulting in an improved fidelity in the process of cellular replication. ${ }^{7}$ P53-regulated gene products interact with many other signal transduction pathways involved in metabolism and growth (PI3K-mTOR pathways), stem cells (the Wnt

\footnotetext{
${ }^{1}$ Simons Center for Sysems Biology, Institute for Advanced Study, Princeton, NJ 08540, USA

*Corresponding author: AJ Levine, Simons Center for Sysems Biology, Institute for Advanced Study, Bloomberg Hall, Einstein Drive, Princeton, NJ 08540, USA. Tel: +1 609734 8005; Fax: +1 609951 4438; E-mail: alevine@ias.edu
} 
pathway), and development affecting the p53-Hippo pathways, ${ }^{7}$ the immune system, the fidelity of epigenetic signals, senescence, aging and life span, or death (reviewed in Wu and Prives $^{8}$ ). It appears that p53-mediated functions are involved from early development, through life, and in death. What is remarkable about this is that the p53 gene and its protein are not essential for life, development, or death under normal circumstances. It is difficult to go through life without stress, however, and many types of stress activate p53, improving fidelity under conditions that would otherwise result in mutations and abnormalities. The p53 protein ensures a quality of life or fidelity either by repair or by death.

The earliest progenitors of the p53 family of genes (Tp53, Tp63, and Tp73), which encode transcription factors, were first observed in evolutionary development in the choanoflagellates, representing one of the earliest multicellular organisms. The evolutionary progeny of these genes have been studied in sea anemones, insects, and worms, where the p53 family of proteins reside in germ cells responding to stresses (such as DNA damage) with apoptotic death, ensuring fidelity of the germ line. In clams, the Tp53 gene is often mutated in leukemias, initiated by movements of retrotransposons throughout the genome (genomic instability) and spread as clones of these cells infect one clam and its neighbors. In vertebrates, p53 specializes in tumor suppression in somatic cells. ${ }^{5,6}$ P63 specializes in the fidelity of the female germ line and as a stem cell transcription factor producing squamous cells in the body. The absence of p63 results in the loss of the skin barrier, resulting in dehydration and infections. The p73 transcription factor has an essential role in producing multiciliated cells in epithelial cell sheets (reviewed in Nemajerova et al. ${ }^{9}$ ) moving germ cells toward fertilization, keeping airways clear in the lungs, and moving fluids in the brain. The absence of p73 function results in sterility and inflammation, as microorganisms are no longer cleared from the airways. The Tp53 family of genes is an important part of our defenses against infectious organisms as barrier functions (p63 and p73) and the innate immune system (p53).

Thus, the Tp53 family of genes has its origins insuring the fidelity of the germ line in invertebrates. Many invertebrates undergo active cell division during multiple stages of development, but become post-mitotic (except in the germ line) as reproducing adults. The vertebrate body plan changed this by using a variety of stem cells to regenerate tissues throughout development and adult life. Although this provided longer adult lifespans, the organism became vulnerable to cancers, and the functions of the Tp53 gene were repurposed from the germ line to the somatic tissues, where tumor suppression became an important set of functions. ${ }^{5,6}$ P53 loss-of-function mutations ${ }^{1}$ became the most common feature of cancers, and gains of function by mutant p53 proteins ${ }^{2}$ contribute to the cancerous phenotype. The Tp53 family of genes in vertebrates never quite lost its functions in the germ line: p63 fidelity of egg production; p73 - motility of germ cells ${ }^{9}$ and, in early development, p53 - implantation of the embryo. In vertebrates, this family of transcription factors has a critical role in protecting the host skin's protective barrier, inducing the innate immune response, preventing movement of retrotransposons in the genome and genomic instability, hypoxia, mitochondrial functions, regulating metabolic pathways and glucose and glutamine utilization, regulating the rates of cell division, maintaining epigenetic stability, monitoring centrosome fidelity and chromosomal segregation (aneuploidy), monitoring and inhibiting viral and bacterial infections, and many other responses to our environment. ${ }^{3-7,9}$ Hidden in some of these functions are the processes of aging and death. ${ }^{8}$ From germ line development, through fertilization, embryonic development, epigenetic programing, birth, organismic development, reproduction, aging, and death, the Tp53 family of genes, first observed over a billion years ago, has an important role throughout our lives by providing fidelity in spite of stress. Elucidating these functions and their coordination by a small family of gene products is the challenge for the future. These reviews point us in some of those directions.

\section{Conflict of Interest}

The author declares no conflict of interest.

\footnotetext{
1. Baugh $\mathrm{E}$ et al. Cell Death Differ 2018; 25: 154-160.

2. Kim MP et al. Cell Death Differ 2018; 25: 161-168.

3. Engeland K. Cell Death Differ 2018; 25: 114-132.

4. Sullivan KD et al. Cell Death Differ 2018; 25: 133-143.

5. Kaiser AM, Attardi LD. Cell Death Differ 2018; 25: 93-103.

6. Aubrey BJ et al. Cell Death Differ 2018; 25: 104-113.

7. Furth $\mathrm{N}$ et al. Cell Death Differ 2018; 25: 81-92.

8. Wu D, Prives C. Cell Death Differ 2018; 25: 169-179.

9. Nemajerova A et al. Cell Death Differ 2018; 25: 144-153.
} 